\title{
Results after mitral valve replacement with cloth- covered Starr-Edwards prostheses (models 6300, 6310/6320, and 6400)
}

\author{
ROBERT FORMAN, WALTER BECK, AND CHRISTIAAN N. BARNARD
}

From The Cardiac Clinic, Groote Schuur Hospital, and Departments of Medicine and Surgery, University of Cape Town, South Africa

SUMMARY The actuarial survival and thromboembolic rates for the three types of cloth-covered StarrEdwards mitral prostheses, models 6300,6310/6320, and 6400 followed 6, 5, and 2 years, respectively, were not significantly different throughout the years they were followed. The combined cumulative survival and thromboembolic proportion at 5 years for these prostheses were 71 and 66 per cent, respectively. The thromboembolic rates were not different in the following two groups : (a) 238 patients receiving anticoagulants, and (b) 52 patients who had discontinued or who were not receiving anticoagulants.

Four patients thrombosed their mitral prostheses. Another 8 per cent had class 3 symptoms after operation, which were attributed to myopathic or restrictive left ventricular dysfunction or other valvular disease.

The incidence of complications, particularly thromboembolism, has significantly decreased since the introduction of cloth covering of the Starr-Edwards mitral prostheses (Bonchek and Starr, 1975). Model 6300 is the earliest model in which both ring and cage were 'teflon' covered. In the 'composite seat' prostheses, models 6310 and 6320 , studs were added to the inner ring to prevent wear of the seat covering by the ball. The current 'track valve', model 6400 , has a metal inner aspect of the polypropylene covered struts to prevent sticking of the ball (Bonchek et al., 1974).

The purpose of this report is to analyse our results after mitral valve replacements with the different cloth-covered Starr-Edwards ball prostheses.

\section{Clinical material and methods}

A total of 332 adult patients had isolated mitral valve replacement, with or without tricuspid annuloplasty, with cloth-covered Starr-Edwards prostheses between January 1970 and June 1976; they were followed until November 1976: 27 with model 6300,180 with models $6310 / 6320$, and 125 with model 6400. During this period 180 additional patients received other mitral prostheses. Selection of prosthesis used was arbitrary and not randomised or consecutive. Three hundred and eighteen patients were in either NYHA functional class 3 or 4, Received for publication 25 May 1977 assessed while receiving therapy with both digitalis and diuretics. Anticoagulant therapy with warfarin was prescribed in all patients starting on the third postoperative day and continued for one month. It was discontinued in patients where the distance they had to travel made it impractical, while specific contraindications were present, or where patients were unreliable. Warfarin therapy was prescribed with the object of maintaining the prothrombin time between $1 \frac{1}{2}$ and $2 \frac{1}{2}$ times control. Quick prothrombin time was measured using local human brain thromboplastin standardised against British comparative thromboplastin. Events considered to be embolic were included in analysis when definite symptoms or signs persisted for 10 minutes or sudden loss of consciousness occurred. Transient dizziness and vertigo by themselves were not included as they were considered to occur frequently o on a non-organic basis. Embolic events were docu- N mented as either definite or possible and only స్ట definite events were considered in analyses. Neuro- 0 logical deficit or embolic episodes were not included

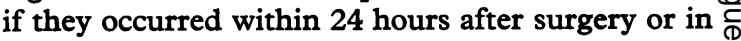
the presence of infective endocarditis.

Actuarial methods, described by Anderson et al. $\square$ (1974), were used to analyse survival and thromboembolic complications. Statistical differences $\stackrel{\mathbb{D}}{\Omega}$ between cumulative thromboembolic or survival $\mathbb{\oplus}$ proportions were estimated by the $\chi^{2}$ test. Operative $\frac{O}{\sigma}$ mortality included all patients dying before dis- 
charge from hospital or within 30 days after surgery. Survival curves include operative mortality. The postoperative status of patients was personally checked at the follow-up clinic by one of us (R.F.), except for patients living in other centres when questionnaires completed by their physicians were used. Nineteen patients were lost to follow-up, 3 of whom were not receiving anticoagulants.

Sixteen patients who had class 3 or 4 symptoms postoperatively were subjected to routine right and left heart haemodynamic studies to determine the cause of their disability. Ejection fraction was measured by the method of Sandler and Dodge (1968) and end-diastolic volume was calculated by dividing cardiac output by the product of heart rate and ejection fraction in the absence of aortic or mitral regurgitation.

\section{Results}

\section{MORTALITY}

\section{Early}

The operative and hospital mortality or the mortality within 30 days of operation for all patients was 8 per cent: 11 per cent for model 6300,9 per cent for model 6310/6320, and 6 per cent for model 6400 prostheses. In no instance did death result from primary prosthetic valve malfunction.

\section{Late}

Thirty-nine late deaths were recorded. Necropsies were performed in 20 , including 4 patients who, despite receiving anticoagulants, died of valvar thrombosis. The commonest cause of death was thromboembolism which was recorded in 15 patients. Two of these patients were not receiving anticoagulants. Nine patients died suddenly and unexpectedly. It is possible that some of these patients had malignant arrhythmias or thromboembolism. Actuarial analysis of survival, which includes operative deaths, for each type of prosthesis is shown in Fig. 1. The 5-year survival of the 332 patients, 82 of whom were in or had completed their fifth year of follow-up, was 71 per cent. There was no significant difference in survival proportions for each model prosthesis at each yearly interval $(P>0 \cdot 1)$, i.e. for 2 years of follow-up of model 6400 and 5 years of models 6300 and $6310 / 6320$.

\section{THROMBOEMBOLISM}

Fifty-three patients developed thromboembolic events, 8 of whom had more than one episode; 5 of these latter patients have had their prostheses removed and replaced with tissue valves. Twenty-five patients had major emboli: 10 died, 5 have been left with permanent (disabling) neurological deficit,

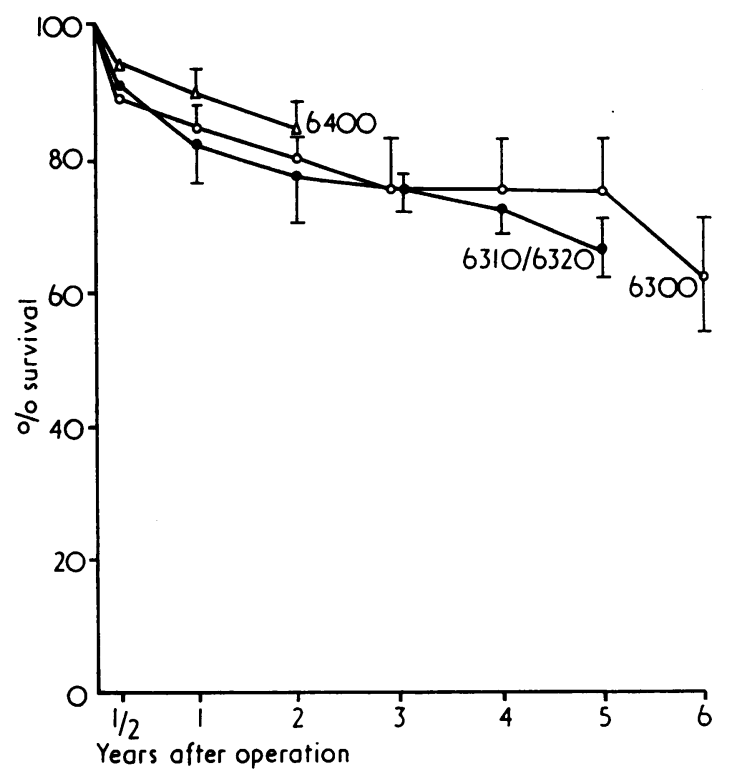

Fig. 1 Actuarial survival curves for three models of cloth-covered Starr-Edwards prostheses. There was no significant difference in interval survival proportions between each model at each year after operation $(P>0.1)$ (vertical bars denote $S E$ ).

and 10 recovered completely after hospital admission. Four additional patients developed valvar thrombosis, and in 2 it was associated with major cerebral amboli. Actuarial analysis of thromboembolic-free events in patients with each type prosthesis is shown in Fig. 2. Only the first occurrence of an event was considered in the analysis (Anderson et al., 1974). There was no significant difference in thromboembolic-free cumulative proportion for each type of prosthesis at each yearly interval $(P>0 \cdot 1)$, i.e. for 2 years of follow-up of model 6400 and 5 years of models 6300 and $6310 /$ 6320. The 5 year cumulative thromboembolic-free proportion for the combined data of the 3 models was 66 per cent.

To determine the effect of anticoagulant therapy after discharge from hospital the data from 3 models were pooled and divided into 2 groups: (a) 238 patients receiving anticoagulant therapy; (b) 52 patients who had discontinued or were not taking anticoagulants.

Thirty patients in group a, despite receiving anticoagulants, developed thromboembolic events in the first 5 years after the first postoperative month: 24 of these patients had their anticoagulation control at our institution and 20 of their prothrombin charts were available for scrutiny. In only 
4 of these patients was the prothrombin time recorded as less than $1 \frac{1}{2}$ times normal in the 6-month period before embolism. One patient in group a died of a cerebral haemorrhage and 22 instances of anticoagulant haemorrhage were recorded. Nine patients in group b were recorded as having thromboembolic episodes during a similar 5-year postoperative period. Actuarial analysis of thromboembolic episodes in these 2 groups is shown in Fig. 3. There was no significant difference between the two curves $(P>0 \cdot 1)$. It was concluded that in our patients anticoagulant therapy was ineffective in preventing thromboembolism.

\section{HAEMODYNAMICS}

Of the surviving 304 patients $24(8 \%)$ had class 3 or 4 symptoms in the postoperative period despite digitalis and diuretic therapy. (Four additional patients developed acute pulmonary oedema and died of valvar thrombosis.) Sixteen of these symptomatic patients were catheterised postoperatively. No evidence of mitral valve malfunction was detected; mean diastolic transvalvar pressure difference was $8 \mathrm{mmHg}$ (range 4 to $14 \mathrm{mmHg}$ ) and mean effective mitral valve area was $1.7 \mathrm{~cm}^{2}$ (range 1.3 to $2.7 \mathrm{~cm}^{2}$ ). No patient had significant paravalvar leak.

Cardiac disability was attributed to the following three types of pathology.

\section{(1) Myopathic left ventricle}

Seven patients were found to have myopathic left ventricles with ejection fractions ranging from $0 \cdot 13$ to 0.32 (mean 0.28 ). Left ventriculograms showed uniform hypokinesis and coronary arteriograms recorded in 5 of these patients were normal. Three of these patients originally had pure mitral stenosis having all previously had mitral valvotomies.

\section{(2) Restrictive left ventricle}

In 4 patients a non-compliant left ventricle was considered to be present because of, (i) raised left ventricular end-diastolic pressure (range 18 to 24 $\mathrm{mmHg}$ ) with dip and plateau pressure tracings; (ii) normal left ventricular contraction with ejection fraction range of 0.62 to 0.68 , and (iii) normal left ventricular volume (range 60 to $78 \mathrm{ml} / \mathrm{m}^{2}$ ). These patients were studied 2 to 6 years postoperatively.

\section{(3) Other valvar disease}

(a) Gross tricuspid regurgitation was the only major significant abnormality detected in 2 patients. Both patients had mild pulmonary hypertension and have not materially benefited from subsequent tricuspid valve replacement.

(b) Severe aortic valve disease, which was not

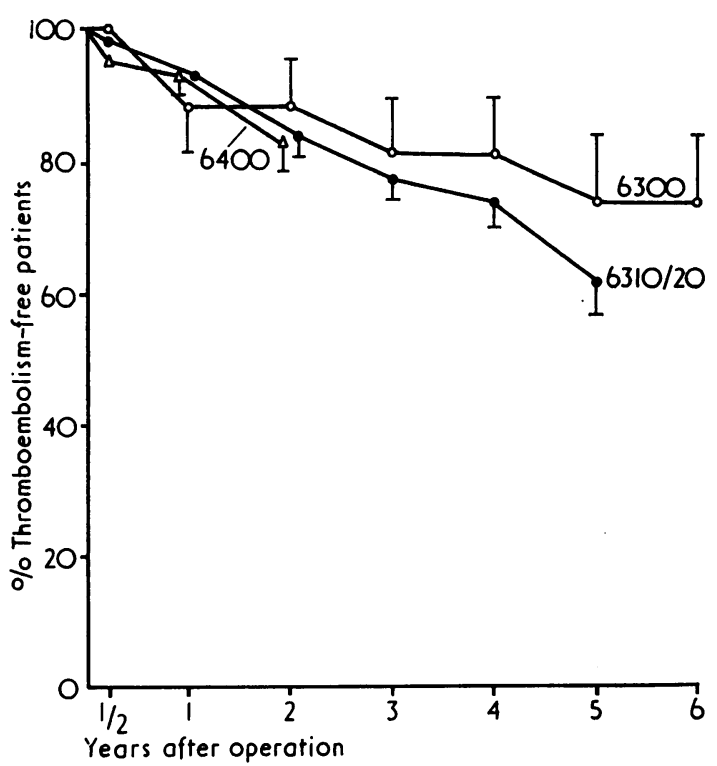

Fig. 2 Actuarial thromboembolism-free curves for 3 models of cloth-covered Starr-Edwards prostheses. There was no significant difference between each model at each year after operation $(P>0 \cdot 1)$ (vertical bars denote $S E)$.

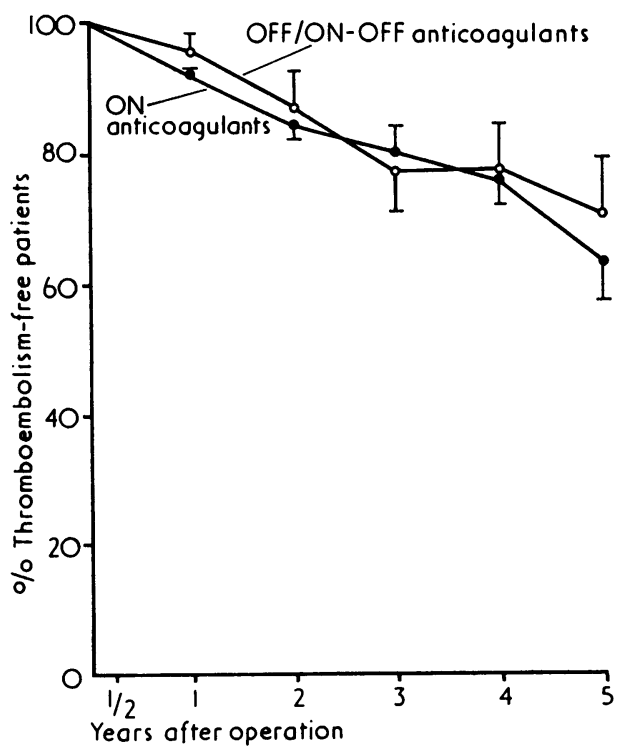

Fig. 3 Actuarial thromboembolism-free curves for patients receiving anticoagulants and those who were not receiving or had discontinued their anticoagulants. There was no significant difference between each curve at each year after operation $(P>0.1)$ (vertical bars denote $S E$ ). 
regarded as significant at their preoperative catheterisation or during surgery, occurred in 3 patients. All have benefited from aortic valve replacement.

\section{Discussion}

The actuarial survival and thromboembolic rates for the three types of cloth-covered Starr-Edwards mitral prosthetic valves, models $6300,6310 / 6320$, and 6400 were not significantly different. This is in contrast to the results of Starr, who found a difference in survival and thromboembolism between models 6300 and 6310/6320 (Kirklin and Pacifico, 1973). The longest follow-up of patients with model 6400 is at present in the third year and our assessment of this prosthesis is, therefore, preliminary. We postulate that it will not be different from the other models when followed over 5 years. The thromboembolic incidence for models 6300 and $6310 / 6320$ is higher than that reported by Starr's group (Bonchek and Starr, 1975) but appears similar to that reported by others (Winter et al., 1972; Cleland and Molloy, 1973; Aris et al., 1974) though exact comparison with these later reports is difficult because they have not expressed their results in actuarial form (Anderson et al., 1974) and the follow-up is over a shorter period.

Anticoagulant therapy in our hands did not decrease the thromboembolic incidence despite what is considered to be adequate anticoagulation (Katholi et al., 1976) in the majority of those patients who had thromboembolic complications. It is possible that the prothrombin time should be controlled at greater than $1 \frac{1}{2}$ to $2 \frac{1}{2}$ times normal or that the level of control should be more even (Vidne et al., 1973). Despite the fact that other authors have also found that anticoagulant therapy did not significantly reduce the incidence of embolism with other prostheses (Stanford et al., 1972), we are reluctant to discontinue anticoagulants in our patients until others have confirmed our results with clothcovered Starr-Edwards valves.

Starr and his co-workers have indicated that the thromboembolic incidence is maximal in the first year (Bonchek and Starr, 1975) and then diminished rapidly. This was not our experience as we found the incidence did not decrease over 5 years of follow-up.

We have not objectively tested exercise capabilities of our patients postoperatively but have relied on their subjective symptoms to assess their effort tolerance. This can be notoriously unreliable (Selzer and Cohn, 1972) and the 8 per cent of patients recorded in this series with class 3 or more symptoms is possibly an underestimate of the postoperative disability. The figure may more real- istically be 15 per cent as reported by Starr's group (Bonchek et al., 1974).

In no instance was either death or disability attributed to prosthetic valve malfunction other than thromboembolism. Roberts et al. (1973) have described extensive endocardial fibrosis seen at necropsy in patients with Starr-Edwards mitral prostheses which they attributed to altered flow patterns around the caged ball. This pathology may account for the restrictive left ventricular disease seen in 4 of our patients. In contrast the myopathy seen in patients with mitral prostheses has been well documented (Peterson et al., 1967; Hildner et al., 1972) and has been variously attributed to rheumatic involvement, chronic volume overload, intraoperative damage, and postoperative microemboli.

\section{References}

Anderson, R. P., Bonchek, L. I., Grunkemeier, G. E., Lambert, L. E., and Starr, A. (1974). The analysis and presentation of surgical results by actuarial methods. Fournal of Surgical Research, 16, 224-230.

Aris, A., Fast, A. J., Tector, A. J., Flemma, R. J., and Lepley, D. (1974). A comparative study of ball and disc prostheses in mitral valve replacement. Fournal of Thoracic and Cardiovascular Surgery, 68, 335-343.

Bonchek, L. I., Anderson, R. P., and Starr, A. (1974). Mitral valve replacement with cloth-covered composite-seat prostheses. The case for early operation. Fournal of Thoracic and Cardiovascular Surgery, 67, 93-109.

Bonchek, L. I., and Starr, A. (1975). Ball valve prostheses: current appraisal of late results. American fournal of Cardiology, 35, 843-854.

Cleland, J., and Molloy, P. J. (1973). Thromboembolic complications of the cloth-covered Starr-Edwards prostheses No. 2300 aortic and No. 6300 mitral. Thorax, 28, 41-47.

Hildner, F. J., Javier, R. P., Cohen, L. S., Samet, P., Nathan, M. J., Yahr, W. Z., and Greenberg, J. J. (1972). Myocardial dysfunction associated with valvular heart disease. American fournal of Cardiology, 30, 319-326.

Katholi, R. E., Nolan, S. P., and McGuire, L. B. (1976). Living with prosthetic heart valves. Subsequent noncardiac operations and the risk of thromboembolism or hemorrhage. American Heart fournal, 92, 162-167.

Kirklin, J. W., and Pacifico, A. D. (1973). Surgery for acquired valvular heart disease. New England fournal of Medicine, 288, 194-199.

Peterson, C. R., Herr, R., Crisera, R. V., Starr, A., Bristow, J. D., and Griswold, H. E. (1967). The failure of hemodynamic improvement after valve replacement surgery. Annals of Internal Medicine, 66, 1-24.

Roberts, W. C., Bulkley, B. H., and Morrow, A. G. (1973). Pathologic anatomy of cardiac valve replacement: a study of 224 necropsy patients. Progress in Cardiovascular Diseases, $15,539-587$.

Sandler, H., and Dodge, H. T. (1968). The use of single plane angiocardiograms for calculation of left ventricular volume in man. American Heart fournal, 75, 325-334.

Selzer, A., and Cohn, K. (1972). Functional classification of cardiac disease: a critique. American fournal of Cardiology, 30, 306-308. 
Stanford, W., Lindberg, E. F., and Armstrong, R. G. (1972). Implantation of heart valve prostheses without anticoagulants. Fournal of Thoracic and Cardiovascular Surgery, 63, 648-651.

Vidne, B., Shimshon, E., and Levy, M. J. (1973). Thromboembolism following heart valve replacement by prosthesis. Survey among 365 consecutive patients. Chest, 63, 713-717.
Winter, T. Q., Reis, R. L., Luke Glancy, D., Roberts, W. C., ฏ Epstein, S. E., and Morrow, A. G. (1972). Current status C of the Starr-Edwards cloth-covered prosthetic cardiac valves. Circulation, 45 and 46, Suppl. 1, I 14-24.

Requests for reprints to Dr R. Forman, Cardiac Clinic, Groote Schuur Hospital Observatory, 7925, South Africa. 